\title{
EFFECTS OF NANO-WOLLASTONITE ON PHYSICAL AND MECHANICAL PROPERTIES OF MEDIUM-DENSITY FIBERBOARD
}

\author{
Hamid Reza Taghiyari ${ }^{1, \wedge}$, Pezhman Nouri ${ }^{1}$
}

\begin{abstract}
Effects of nano-wollastonite (NW) on physical and mechanical properties of medium density fiberboard (MDF) were studied. NW was applied at 5, 10, 15, and $20 \mathrm{~g} / \mathrm{kg}$ dry weight basis of wood fibers; the results were then compared with control specimens. Two application methods of NW were used: surface and internal applications. Density was kept constant at $0,67 \mathrm{~g} / \mathrm{cm}^{3}$ for all treatments and tests were carried out in accordance with ASTM D-1037. Addition of NW contributed in improving the physical and mechanical properties of the panels, both when applied internally or as surface treatment. It was concluded that NW contents of $10 \%$ and $15 \%$ were the optimal levels for industrial purposes for internal and surface applications, respectively.
\end{abstract}

Keywords: Composite board, fiber/matrix bond, medium-density fiberboard, minerals, nanoscience, thermal properties, Wollastonite.

\section{INTRODUCTION}

Composite-boards have the advantages of a homogeneous structure and the use of residual raw materials. However, water absorption (WA) and thickness swelling (TS) are high in most of woodcomposite panels. Moreover, transfer of heat to the core layer of the mat is a problem, because the thermal conductivity coefficient of wood is low, and addition of water to the mat is limited to some extent to avoid blowing of panels after the hot press. Many studies have been carried out to decrease WA and TS, and find methods for limitation of formaldehyde emission as well as promotion of the quality of the resins (Valenzuela et al. 2012). In this connection, nano-materials can easily be used in composite panels as an in-process treatment (IPT). High thermal conductivity of nano-metals (Saber et al. 2013, Yu et al. 2012) was used to accelerate production rate at industrial scale and to decrease liquid permeability.

Nano-wollastonite, as a silicate mineral $\left(\mathrm{CaSiO}_{3}\right)$, was reported to improve fire-retarding properties in medium-density fiberboards and solid woods (Gallo et al. 2012, Haghighi et al. 2013) as well as to increase biological resistance to wood-deteriorating fungi (Karimi et al. 2013). Application of nanowollastonite demonstrated promising results on fire-retarding properties in medium-density fiberboard (MDF) (Taghiyari et al. 2013a). However, its effects on the physical and mechanical properties of composite panels have not been studied. The present research project was therefore carried out to find out its possible improving effects on the physical and mechanical properties in MDF, both when applied as IPT, and when applied on the surface of the MDF panels.

\footnotetext{
'Wood Science and Technology Department, Faculty of Civil Engineering, Shahid Rajaee Teacher Training University, Lavizan, Shabanloo St., Tehran, Iran.

^Corresponding author: htaghiyari@srttu.edu

Received: 12.11.2014 Accepted: 22.07.2015
} 


\section{MATERIALS AND METHODS}

\section{Specimen Procurement}

Wood fibers were procured from Sanaye Choobe Khazar Company in Iran (MDF Caspian Khazar). The fibers comprised a mixture of five species of beech, alder, maple, hornbeam, and poplar from the neighboring forests. Boards were $8 \mathrm{~mm}$ in thickness, and the density was kept constant at $0,67 \mathrm{~g} / \mathrm{cm}^{3}$. The total nominal pressure of the plate was 160 Bar. The temperature of the plates was fixed at $130^{\circ} \mathrm{C}$. Hot-pressing continued for 8 minutes. Urea-Formaldehyde resin (UF) was procured from Sari Resin Manufacturing Company in Sari, Iran. 10\% of UF with 200-400 cP in viscosity, 47 seconds of gel time, and $1,277 \mathrm{~g} / \mathrm{cm}^{3}$ in density was used. Ten boards were made for each treatment. From each board, two specimens of $15 \mathrm{~cm}$ in length and $13 \mathrm{~cm}$ in width were cut.

\section{Nano-Wollastonite Application}

Nanowollastonite (NW) gel was produced in cooperation with Vard Manufacturing Company of Mineral and Industrial Products, Iran. Compositions and formulations of the NW are detailed in table 1. The size range of nanowollastonite was $30-100 \mathrm{~nm}$. NW was applied in two ways: 1- Mixed with the UF resin and sprayed on to the fiber before the hot press (internal application of NW), 2- Mixed with a water-based paint and sprayed on to the surfaces of the MDF specimens 2 months from the date of their production (surface application of NW); all physical and mechanical tests were carried out also 2 months after spraying of the NW on the surface of the specimens. In both methods of Internal Application (IA) and Surface Application (SA), the consumption levels of NW remained the same (0, 5, 10, 15, and 20\%).

Table 1. The compounds and formulations of the nanowollastonite gel used in the present study (Taghiyari et al. 2014).

\begin{tabular}{cc}
\hline \hline $\begin{array}{c}\text { Nano-Wollastonite } \\
\text { Compounds }\end{array}$ & Mixing ratio (\%) \\
\hline \hline $\mathrm{CaO}$ & 39,77 \\
\hline $\mathrm{SiO}_{2}$ & 46,96 \\
\hline $\mathrm{Al}_{2} \mathrm{O}_{3}$ & 3,95 \\
\hline $\mathrm{Fe}_{2} \mathrm{O}_{3}$ & 2,79 \\
\hline $\mathrm{TiO}_{2}$ & 0,22 \\
\hline $\mathrm{K}_{2} \mathrm{O}$ & 0,04 \\
\hline $\mathrm{MgO}$ & 1,39 \\
\hline $\mathrm{Na}_{2} \mathrm{O}$ & 0,16 \\
\hline $\mathrm{SO}_{3}$ & 0,05 \\
\hline \hline
\end{tabular}

\section{Physical and Mechanical Tests}

Physical and mechanical tests, as well as the number and location of the specimens, were carried out in accordance with the ISIRI 9044 PB Type P2 (comparable to ASTM D-1037) specifications. The static bending test was performed using center-point loading over a $390 \mathrm{~mm}$ span with the load applied in the tangential direction. The loading speed was $2 \mathrm{~mm} / \mathrm{min}$. All tests were conducted using an INSTRON 4486 testing machine. Internal bond was only measured in control and IA-treatments because SA-treatments were comprised the same control boards having NW sprayed on them; that is, surface application of NW was considered not having any significant effect on the IB of the boards. The moisture content of the specimens at the time of testing was 7,5\% because wood has a thermo-hygromechanical behavior, and its properties depends on the combined action of temperature, relative humidity, and mechanical load variations (Figueroa et al. 2012). 
Hardness was measured at 2, 3, and $4 \mathrm{~mm}$ of penetrations in order to evaluate the effect of nanowollastonite on different depth of penetrations. Also, brittleness was calculated by using the ratio $(\%)$ of the work absorbed in the elastic region (area 1) to the total work absorbed to maximum load, that is, the elastic and plastic regions together (area $1+$ area 2) (Phuong et al. 2007, Taghiyari et al. 2013b) (Equation 1).

$$
\text { Brittleness }=\frac{\text { Area } 1}{\text { Area } 1+\text { Area } 2} \times 100(\%)(\text { Equation 1) }
$$

The proportional limit was decided by drawing a linear correlation curve using data from $0 \%$ load to the limit value (correlation coefficient $\mathrm{R}^{2}=0,999$ ).

\section{Statistical Analysis}

Statistical analysis was conducted using SAS software program, version 9.2 (2010). Two-way analysis of variance (ANOVA) was performed on the data to conclude significant differences at the $95 \%$ level of confidence. Hierarchical cluster analysis, including dendrogram and using Ward methods with squared Euclidean distance intervals, was carried out by SPSS/18 (2010) (Ada 2013). Fitted-line plot was made by Minitab software, version 16.2.2 (2010).

\section{RESULTS}

Visual observation showed there was no difference between the control panels and panels with NW contents of 5, 10, and 15\%. Fibers on the surface of panels were somehow loose in the NW-20\% panels, making a rough appearance to them. Surface application of NW resulted in the same roughness on the surface of panels as a result of the water-based paint that was sprayed on the panels; however, the fibers were not loose as the paint helped the fibers being stuck in their position.

NW significantly decreased water absorption and thickness swelling, both when applied internally or as surface treatment (Figure 1 and 2). The lowest water absorption was observed when 10 and 15\% of nanowollastonite was used as surface treatment. Also, the lowest thickness swelling was seen in SA-NW10\%. Internal application of wollastonite had less decreasing impact on water absorption and thickness swelling than that of surface application. The lowest water absorption and thickness swelling in internal application were seen in NW10\%. 


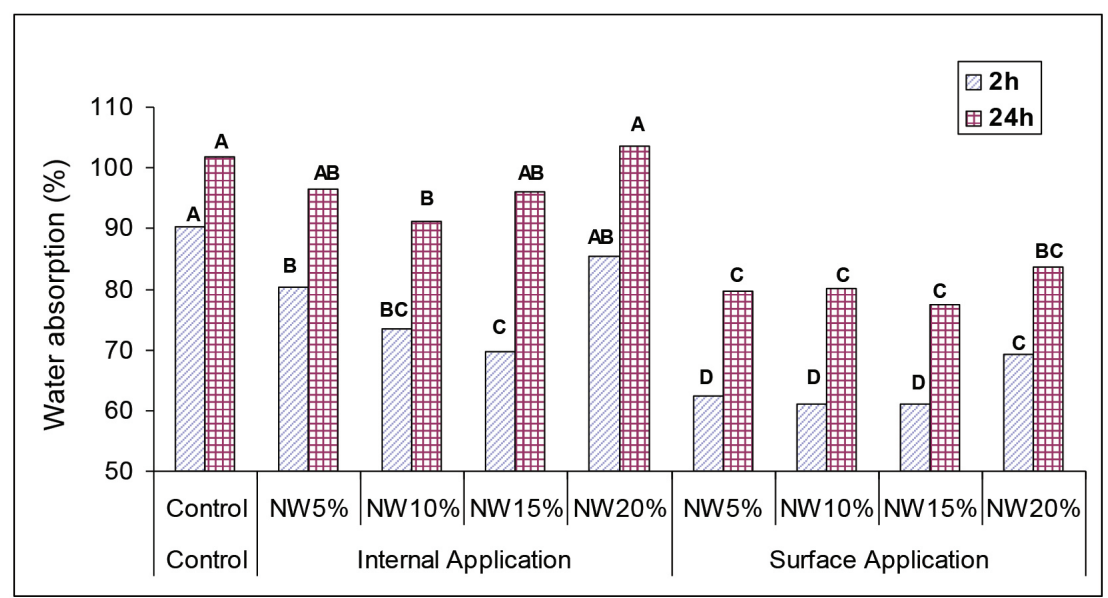

Figure 1. Water absorption (\%) at 2 and 24 hours in the nine different treatments of control, internal and surface applications

(NW = nanowollastonite; 5, 10, 15, and $20=$ percent of nanowollastonite levels), (Letters on the columns are Duncan groupings at $99 \%$ level of confidence).

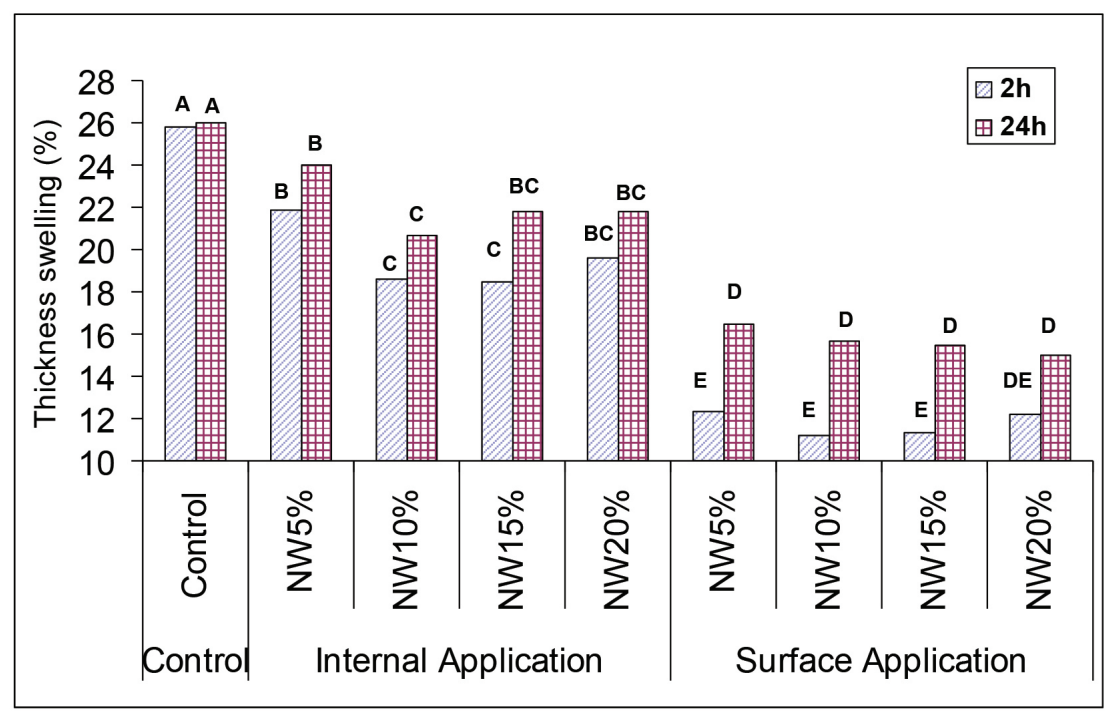

Figure 2. Thickness swelling (\%) at 2 and 24 hours in the nine different treatments of control, internal and surface applications

(NW = nanowollastonite; 5, 10, 15, and $20=$ percent of nanowollastonite levels), (Letters on the columns are Duncan groupings at $99 \%$ level of confidence). 
As to the mechanical properties, NW revealed increasing effects on all the three properties measured. The highest MOR value was observed when 5 percent of NW was used as surface treatment (19,44 MPa) (Figure 3). Surface application of NW also resulted in the highest MOE when used at 20\% (Figure 4). The highest internal bond was found in NW15\%, showing $63 \%$ of increase in comparison to the control specimens (Figure 5).

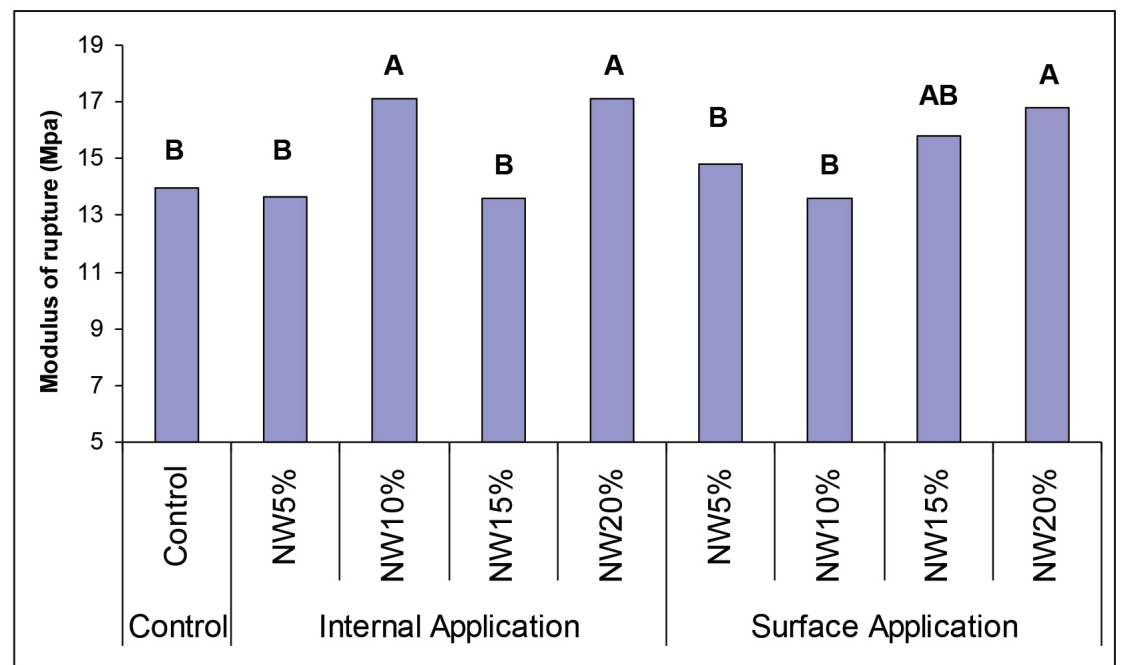

Figure 3. Modulus of rupture in the nine different treatments of control, internal and surface applications

(NW = nanowollastonite; 5, 10, 15, and $20=$ percent of nanowollastonite levels), (Letters on the columns are Duncan groupings at $99 \%$ level of confidence).

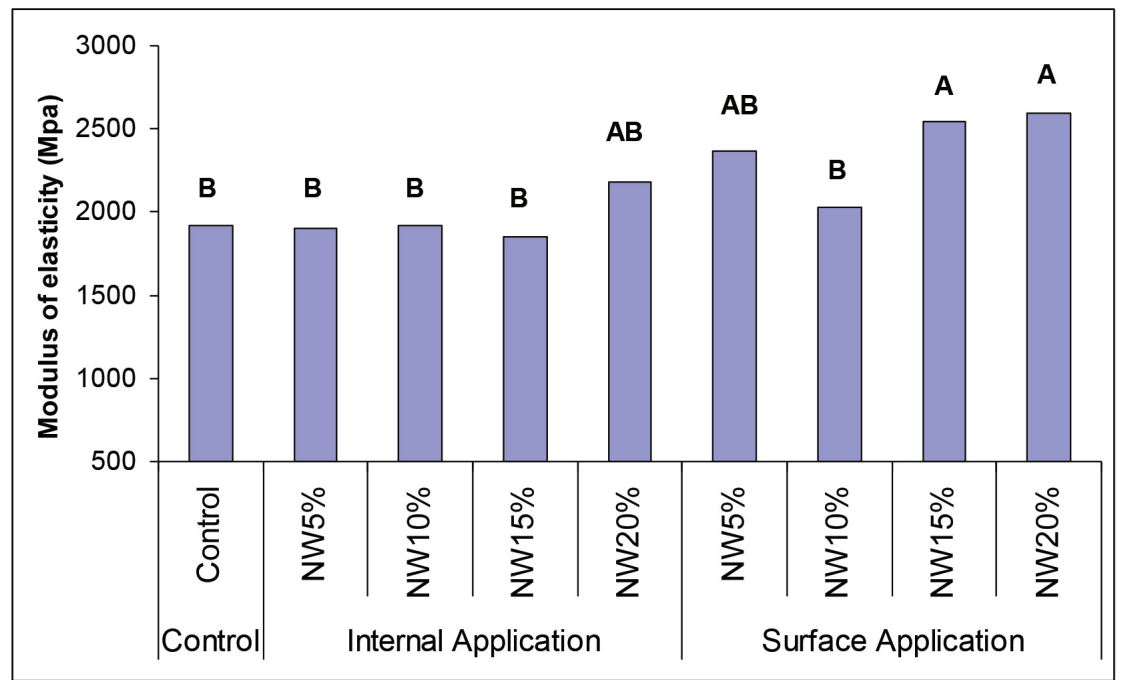

Figure 4. Modulus of elasticity in the nine different treatments of control, internal and surface applications

(NW = nanowollastonite; 5, 10, 15, and $20=$ percent of nanowollastonite levels), (Letters on the columns are Duncan groupings at $99 \%$ level of confidence). 


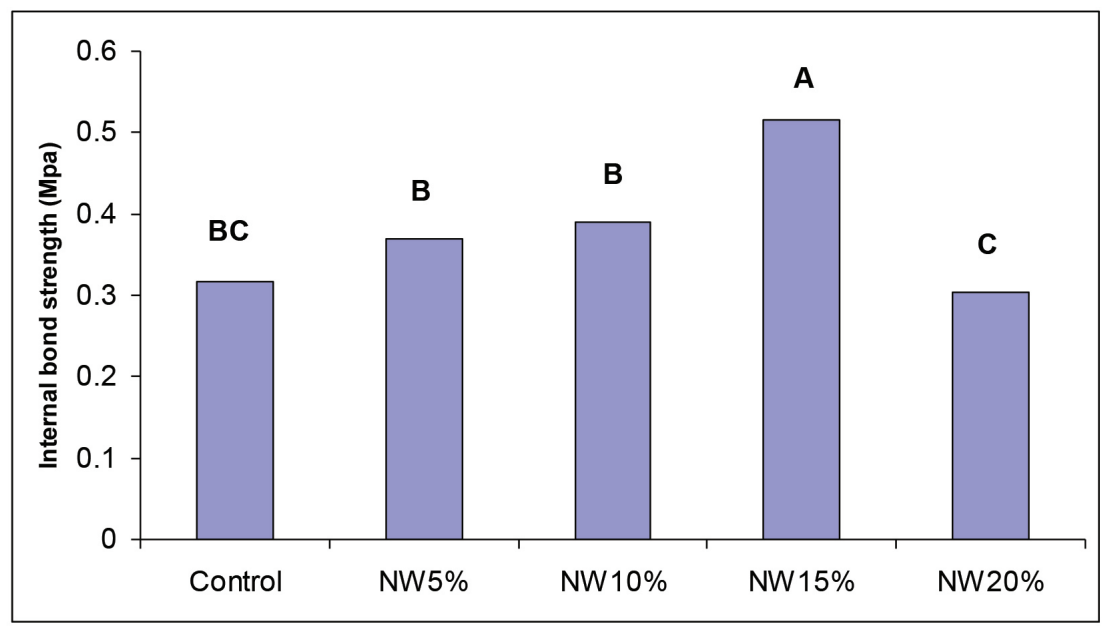

Figure 5. Internal bond in different treatments of control and internal application (NW = nanowollastonite; 5, 10, 15, and $20=$ percent of nanowollastonite levels),

(Letters on the columns are Duncan groupings at 99\% level of confidence).

Hardness was generally improved by adding nanowollastonite both when applied internally or as surface treatment (Figure 6). Similar increasing and decreasing trends were found in the 2, 3, and 4-mm of penetration of the ball in all treatments; however, for reporting purposes, 4-mm of the modified ball penetration was considered here. In the internal fiber treatments, the highest improvement was achieved in NW-5\% treatment $(63,2 \mathrm{MPa})$; and the lowest in NW-20\% $(38,5 \mathrm{MPa})$. In fact, the only treatment showing less hardness values than those in the control treatment was IA-NW-20\%. All four surface fiber treatments showed higher hardness values than those of the control treatment. The highest value in the surface treatments was found in the NW-15\%. Both NW-application methods significantly improved brittleness (Figure 7). NW increased brittleness in IA-NW-15\% to more than $78 \%$ in comparison to the control value.

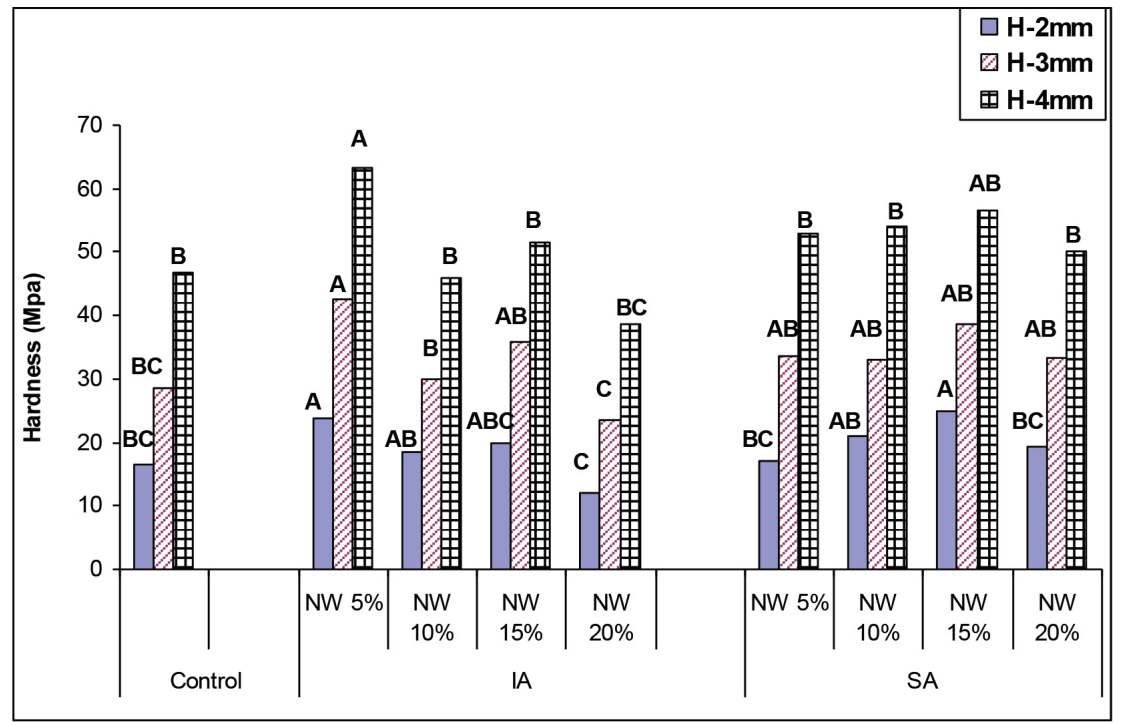

Figure 6. Hardness for 2, 3, and $4 \mathrm{~mm}$ penetration of the modified ball in different treatments of control and internal application

(NW = nanowollastonite; 5, 10, 15, and $20=$ percent of nanowollastonite levels), (Letters on the columns are Duncan groupings at $99 \%$ level of confidence). 


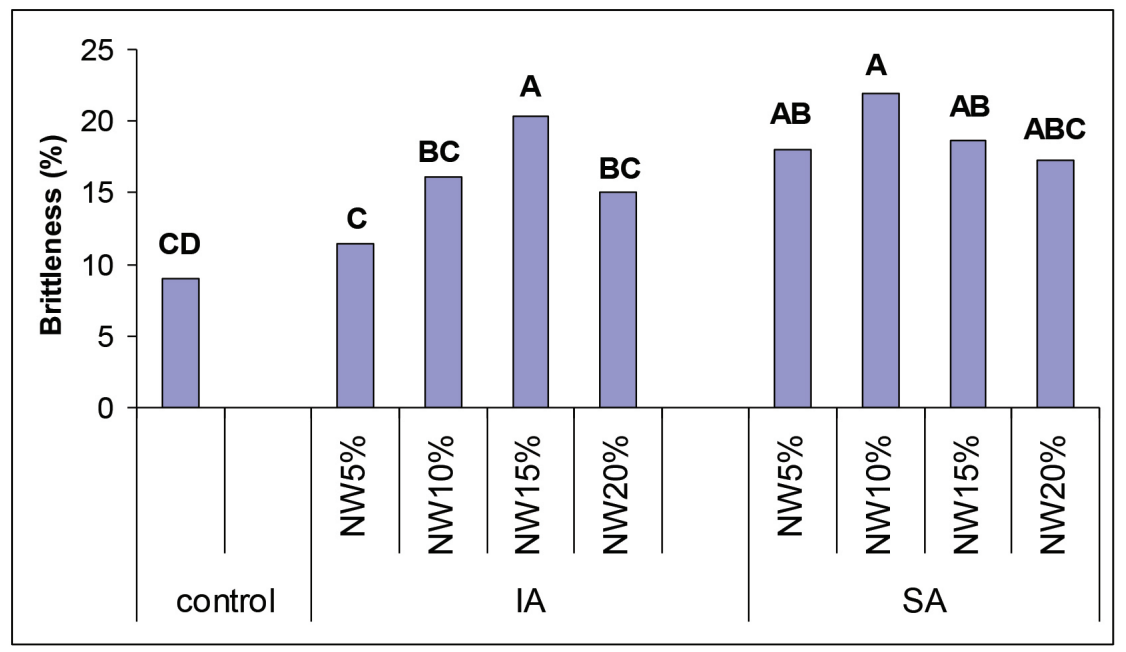

Figure 7. Brittleness in different treatments of control and internal application (NW = nanowollastonite; 5, 10, 15, and $20=$ percent of nanowollastonite levels), (Letters on the columns are Duncan groupings at $99 \%$ level of confidence).

\section{DISCUSSION}

NW showed significant decreasing effect on water absorption and thickness swelling when NW was used up to $15 \%$ in the mat; more than this NW-content level resulted in an increasing trend in WA. The reason may be traced to the equality of the density of different treatments; that is, in the IA-NW20\% boards, less amount of fibers are used and therefore, the integrity and compactness of fibers are less than in other NW-treatments. Thus, the water-repelling property of NW was not enough to prevent the excessive penetration of water in the MDF matrix. Similar increase in permeability was reported in nano-zycosil-treated MDF panels. Furthermore, surface application showed generally lower amounts of water absorption and thickness swelling in comparison to the internal application of NW. This proved that surface protection against penetration of water could be more effective in the short-time tests ( 2 and 24 hours). Still, long-term water absorption and thickness swelling tests should be carried out to have a better scope of the effects of NW on WA and TS. A good correlation was found between thickness swelling versus water absorption (Figure 8), showing a relationship between the amount of water penetrated through the voids and empty spaces among fibers with the amount the fibers were swollen. 


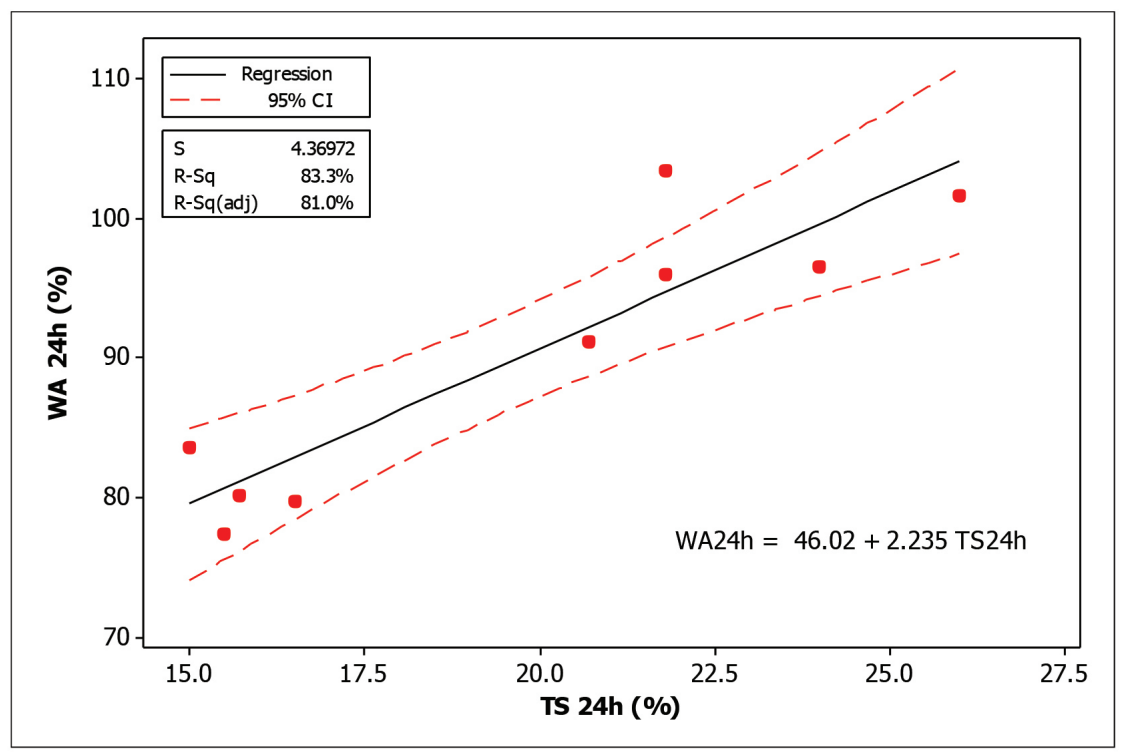

Figure 8. Fitted-line plot between thickness swelling and water absorption after 24 hours immersion in water (WA=water absorption; TS=thickness swelling).

Although NW-application resulted in an increasing trend in the MOR values, but no clear trend could be determined within different consumption levels; nor was it possible between surface and internal applications. However, significant higher values resulted in higher MOE when NW was applied on the surface of the specimens. This showed that the elasticity of the specimens were quite sensitive to their surface quality; that is, NW contributed to the integrity of the surface properties and therefore, MOE values increased with higher NW-contents. This may be better reasoned with due consideration to the highest MOE in SA-NW20\%. NW also improved the internal bond up to $15 \%$ of content. Higher than this amount resulted in a decrease in IB, due to the lower integrity of the fibers resulted from the lower density of fibers in the MDF-matrix as well as absorption of the resin with NW instead of their involvement in the sticking of wood fibers together. Furthermore, an increase in the thermal conductivity coefficient by nano-wollastonite (Taghiyari et al. 2013c) caused better curing of the resin in the core section of the mat, resulting in higher internal bond; similar increase in the internal bond caused by the increase in thermal conductivity by metal nanoparticles ( $\mathrm{Li}$ 2012, Taghiyari 2013, Wegener and Jones 2006) in particleboard. It can be concluded that NW have contributed in forming better bonds between the wood fibers in the MDF-matrix up to $15 \%$; however, higher level $(20 \%)$ would result in lower integrity between the fibers in a way that NW could not compensate for.

NW resulted in a significant increase in the hardness, both when applied internally or as surface treatment. Hardness values of the IA-treatments showed a general decreasing trend as the NW-content increased, due to the lower fiber-content in the MDF-matrix. Hardness at deeper penetration depth of the modified ball (that is, $4 \mathrm{~mm}$ of penetration) showed higher difference between internal treatments; however, surface treatments showed higher difference at lower depth of penetration $(2 \mathrm{~mm})$. Contribution of NW in forming bonds with the wood fibers in the MDF-matrix can also be observed from the brittleness values (Figure 7). 

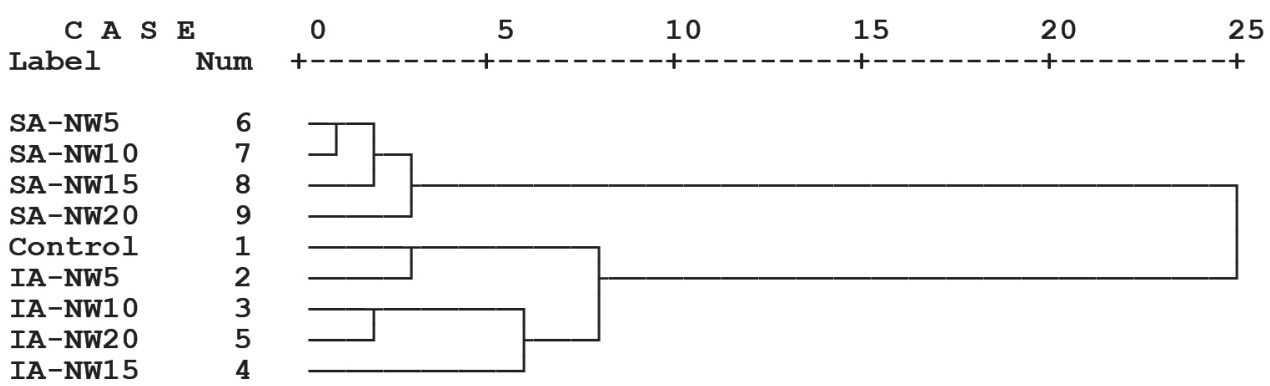

Figure 9. Cluster analysis of the nine treatments of control, surface and internal applications $(\mathrm{IA}=$ internal application; $\mathrm{SA}=$ surface application; $\mathrm{NW}=$ nanowollastonite; 5, 10, 15, and $20=$ percent of nanowollastonite levels).

Cluster analysis of the nine treatments based on all the physical and mechanical properties clearly showed that the surface and internal applications are clustered differently (Figure 9). This proves the significant higher impact of surface over internal application on physical and mechanical properties. An internal NW5\% application was closely clustered with the control specimens; this indicated that internal level of $5 \%$ was not enough to significantly improve the properties when applied internally. The results obtained from IA-NW-10 and 20\% were rather similar. In this connection, two mechanisms acted simultaneously: NW improved the properties in the NW-20\%, however, lower wood-fiber-integration and formation of micro-cavities in the MDF-matrix resulted in a significant decrease in the properties; consequently, the end-results were somehow comparable to those of the NW-10\% panels. These micro-cavities were reported to have negative effects on fire-retarding properties in MDF (Taghiyari et al. 2013a). As to the surface application of NW, the cluster analysis clearly showed that all levels of surface applied NW had significant improving effects on physical and mechanical properties. SA-NW5 and $10 \%$ were clustered closely showing their similarity. However, the best properties were obtained when 15 and $20 \%$ of NW were used surface applied. It can therefore be concluded that NW-15\% can be recommended to decrease the production costs when applied on the surface.

\section{CONCLUSIONS}

NW contributes in making bonds between the wood fibers and consequently improve physical and mechanical properties; it can therefore be recommended in the manufacturing of MDF to improve physical and mechanical properties.

NW content of 5\% is not enough to significantly improve the properties; also, NW20\% is too much and decreases the properties due to the lower amount of the wood fiber used, and the consequent lower integration of wood fibers in the MDF-matrix, when the density of the boards is kept constant;

NW-content of $15 \%$ can be recommended to improve the physical and mechanical properties both for surface and internal applications.

\section{ACKNOWLEDGMENTS}

We appreciate the scientific support of Mr. Jack Norton (retired, Horticulture \& Forestry Science, Queensland Dept. of Agriculture, Australia). 


\section{REFERENCES}

Ada, R. 2013. Cluster analysis and adaptation study for safflower genotypes. Bulgarian Journal of Agricultural Science 19(1): 103-109.

American Society for Testing and Materials. ASTM. 2007. Standard methods for evaluating properties of wood-base fiber and particle panel materials. ASTM D 1037-96a. Philadelphia

Figueroa, M.; Bustos, C.; Dechent, P.; Reyes, L.; Cloutier, A.; Giuliano, M. 2012. Analysis of rheological and thermo-hygro-mechanical behaviour of stress-laminated timber bridge deck in variable environmental conditions. Maderas: Ciencia y tecnologia 14:303-319.

Gallo, E.; Schartel, B.; Acierno, D.; Cimino, F.; Russo, P. 2012. Tailoring the flame retardant and mechanical performance of natural fiber-reinforced biopolymer by multi-component laminate. Composites: Part B 44:112-119.

Haghighi-Poshtiri, A.; Taghiyari, H.R.; Karimi, A.N. 2013. The optimum level of nano-wollastonite consumption as fire-retardant in poplar wood (Populus nigra). International Journal of Nano Dimension 4(2): $141-151$.

Karimi, A.; Taghiyari, H.R.; Fattahi, A.; Karimi, S.; Ebrahimi, Gh.; Tarmian, A. 2013. Effects of wollastonite nanofibers on biological durability of poplar wood (Populus nigra) against Trametes versicolor. BioResources 8(3): 4134-4141.

Li, D. 2012. Nanostructuring materials towards conventionally unachievable combination of desired properties. Journal of Nanomaterials \& Molecular Nanotechnology 1(1): dx.doi.org/10.4172/2324-8777.1000e102.

Phuong, L.X.; Shida, S.; Saito, Y. 2007. Effects of heat treatment on brittleness of Styrax tonkinensis wood. $J$ Wood Sci 53:181-186.

Saber, R.; Shakoori, Z.; Sarkar, S.; Tavoosidana, Gh.; Kharrazi, Sh.; Gill, P. 2013. Spectroscopic and microscopic analyses of rod-shaped gold nanoparticles interacting with single-stranded DNA oligonucleotides. IET Nanobiotechnol 7:42-49

Taghiyari, H.R. 2013. Effects of heat-treatment on permeability of untreated and nanosilver-impregnated native hardwoods. Maderas Ciencia y tecnologia 15(2): 183-194.

Taghiyari, H.R.; Rangavar, H.; Nouri, P. 2013a. Fire-retarding properties of nanowollastonite in MDF. European Journal of Wood and Wood Products 71(5): 573-581.

Taghiyari, H.R.; Enayati, A.; Gholamiyan, H. 2013b. Effects of nano-silver impregnation on brittleness, physical and mechanical properties of heat-treated hardwoods. Wood Science and Technology 47(3): 467-480.

Taghiyari, H.R.; Mobini, K.; Sarvari-Samadi, Y.; Doosti, Z.; Karimi, F.; Asghari, M.; Jahangiri, A.; Nouri, P. 2013c. Effects of nano-wollastonite on thermal conductivity coefficient of medium-density fiberboard. Journal of Nanomaterials and Molecular Nanotechnology 2:1 http://dx.doi.org/10.4172/2324-8777.1000106.

Taghiyari, H.R.; Bari, E.; Schmidt, O.; Tajick-Ghanbary, M.A.; Karimi, A.; Tahir, P.M.D. 2014. Effects of nanowollastonite on biological resistance of particleboard made from wood chips and chicken feather against Antrodia vaillantii. International Biodeterioration \& Biodegradation 90: 93-98.

Valenzuela, J.; Von Leyser, E.; Pizzi, A.; Westermeyer, C.; Gorrini, B. 2012. Industrial production of pine tannin-bonded particleboard and MDF. European Journal of Wood and Wood Products 70(5):735-740.

Wegner, T.H.; Jones, P.H.E. 2006. Advancing cellulose-based nanotechnology. Cellulose 13: 115-118.

Yu, Y.; Jiang, Z.; Wang, G.; Tian, G.; Wang, H.; Song, Y. 2012. Surface functionalization of bamboo with nanostructured ZnO. Wood Science and Technology 46:781-790. 